Supporting Information

\title{
Lightweight and Flexible Bi@Bi-La Natural Leather Composites with Superb X-ray Radiation Shielding Performance and Low Secondary Radiation
}

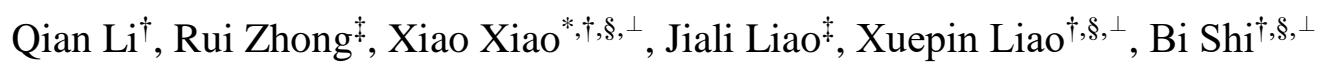

$\dagger$ College of Biomass Science and Engineering, Sichuan University, Sichuan 610065, China

$\$$ Key Laboratory of Radiation Physics and Technology, Ministry of Education, Sichuan University, Sichuan 610065, China

$\S$ National Engineering Research Center of Clean Technology in Leather Industry, Sichuan University, Sichuan 610065, China

$\perp$ Key Laboratory of Leather Chemistry and Engineering, Ministry of Education, Sichuan University, Sichuan 610065, China

Corresponding Author’s E-mail: xiao_xiao@scu.edu.cn.

\section{This document includes:}

Figure S1 to S15 and Table S1 to S4 


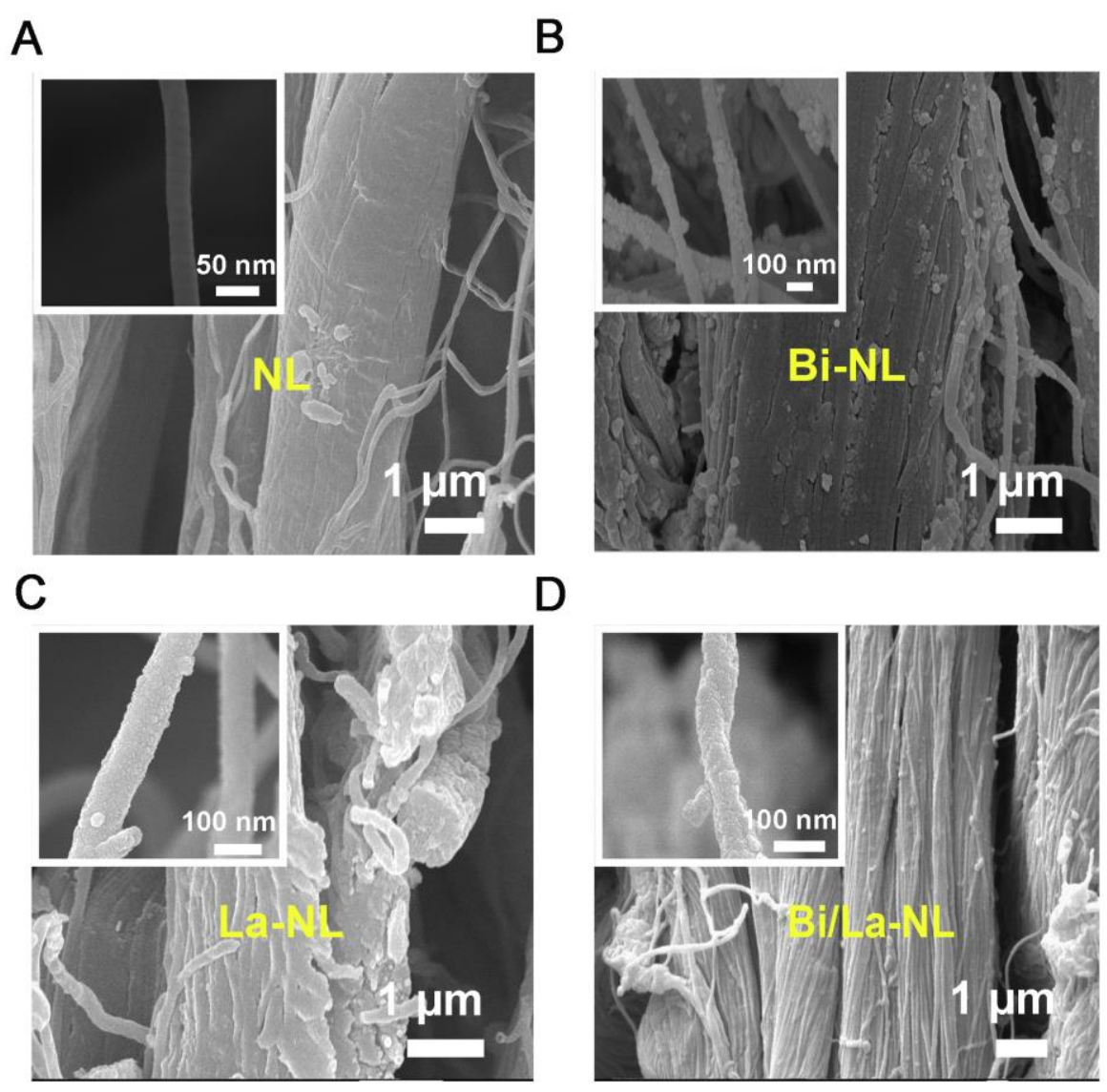

Figure S1. SEM image of NL, Bi-NL, La-NL and Bi/La-NL. 

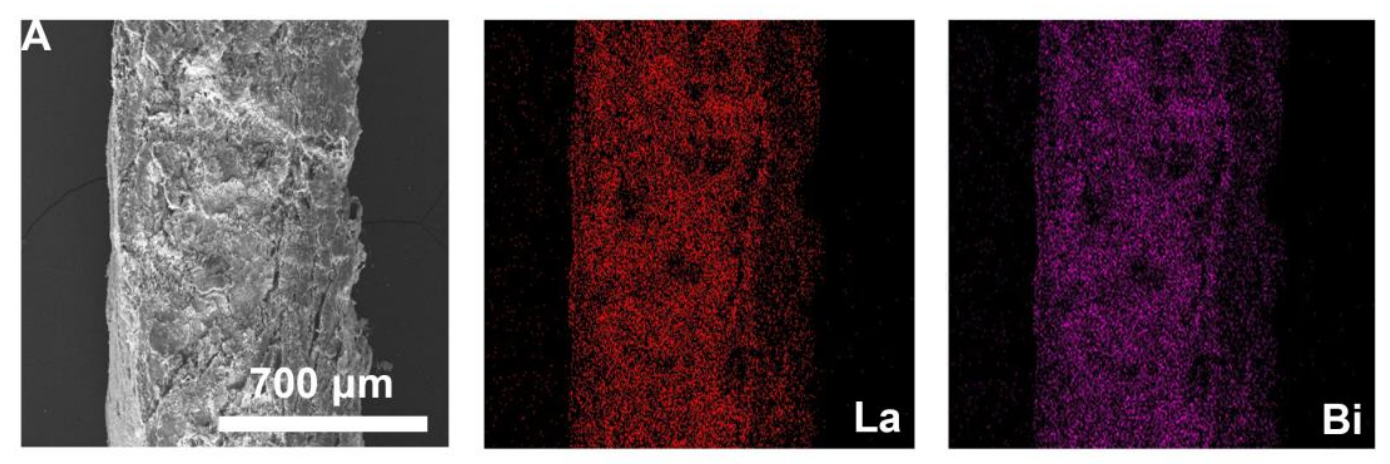

Figure S2. EDX Mapping image of $\mathrm{Bi}_{1.51} \mathrm{La}_{1.51} \mathrm{NL}$. 

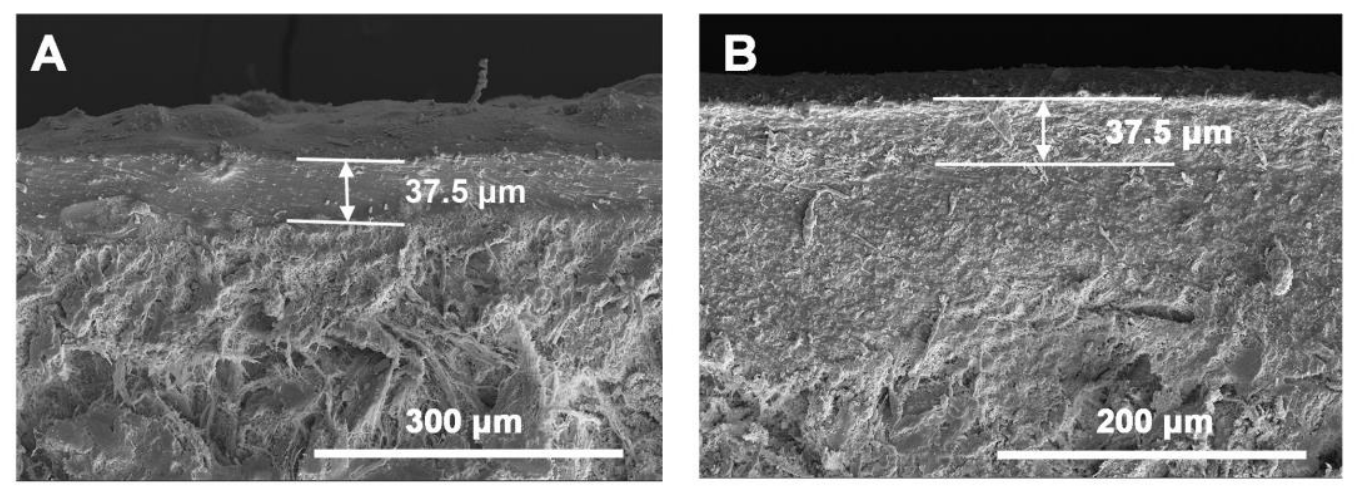

Figure S3. (A) SEM of $\mathrm{La}_{28.2} @ \mathrm{Bi}_{3.48} \mathrm{La}_{3.48}-\mathrm{NL}$. (B) SEM of Bi $28.2 @ \mathrm{Bi}_{3.48} \mathrm{La}_{3.48}-\mathrm{NL}$. 

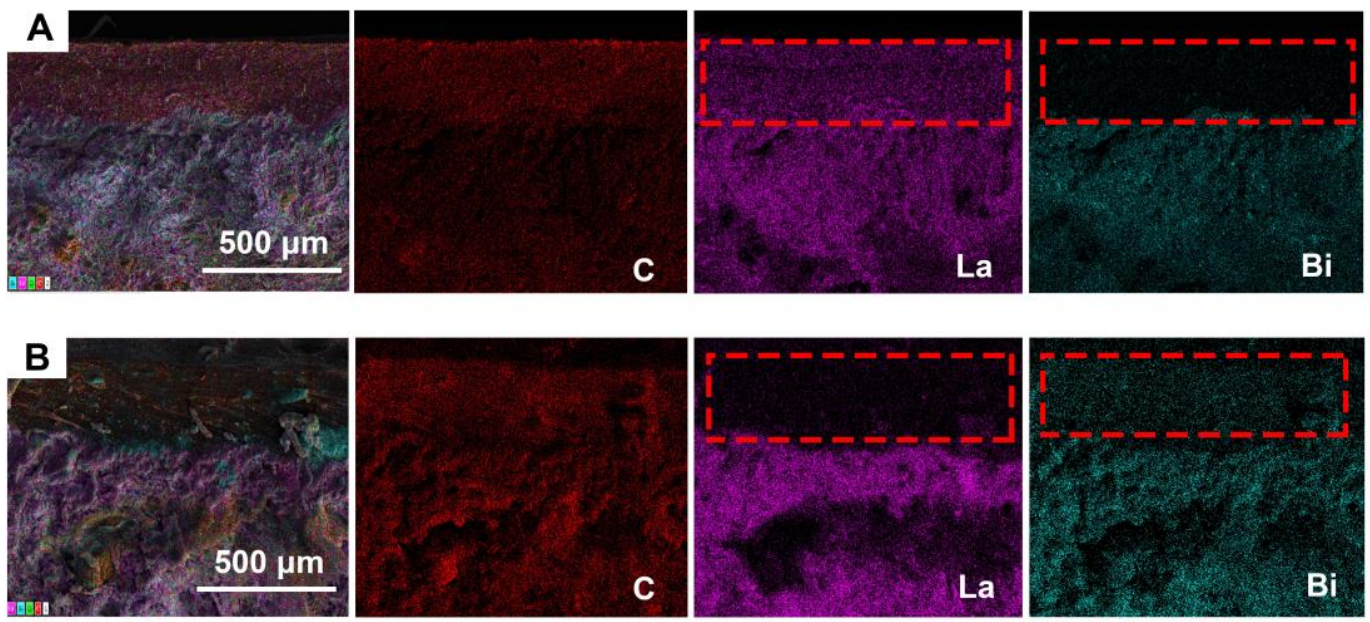

Figure S4. (A) EDX Mapping of La28.2@ Bi3.48La3.48-NL. (B) EDX Mapping of $\mathrm{Bi}_{28.2} @ \mathrm{Bi}_{3.48} \mathrm{La}_{3.48}-\mathrm{NL}$. 


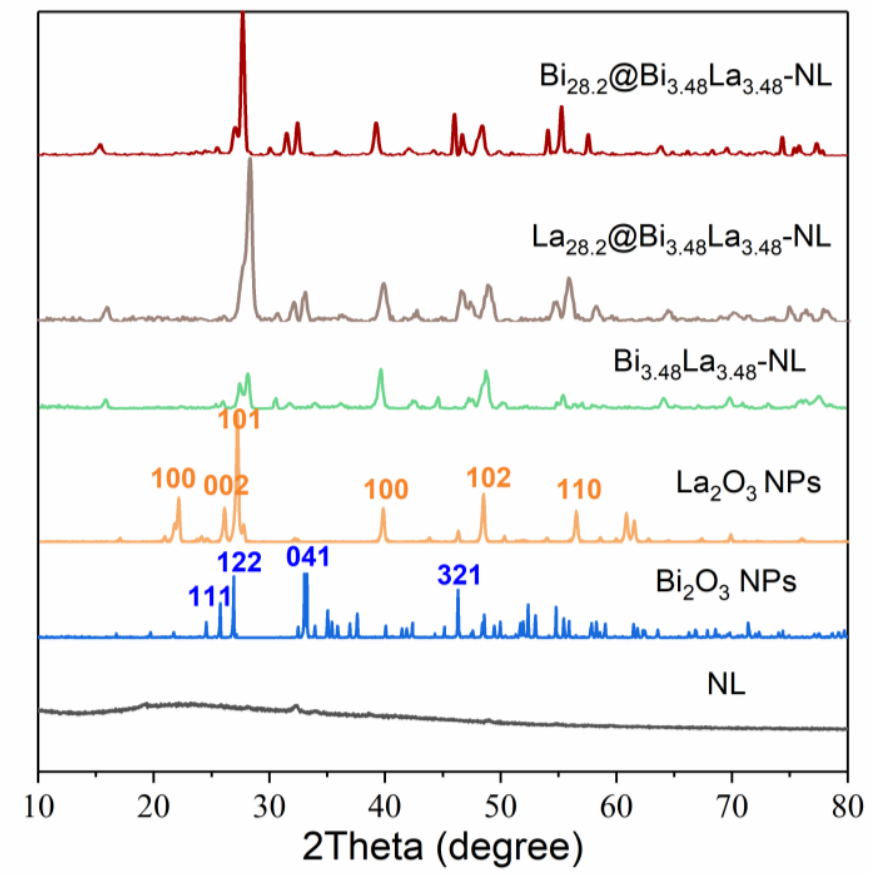

Figure S5. XRD of NL, $\mathrm{Bi}_{2} \mathrm{O}_{3} \mathrm{NPs}, \mathrm{La}_{2} \mathrm{O}_{3} \mathrm{NPs}, \mathrm{Bi}_{3.48} \mathrm{La}_{3.48}-\mathrm{NL}, \mathrm{La}_{28.2} @ \mathrm{Bi}_{3.48} \mathrm{La}_{3.48}-\mathrm{NL}$ and $\mathrm{Bi}_{28.2} @ \mathrm{Bi}_{3.48} \mathrm{La}_{3.48}-\mathrm{NL}$. 


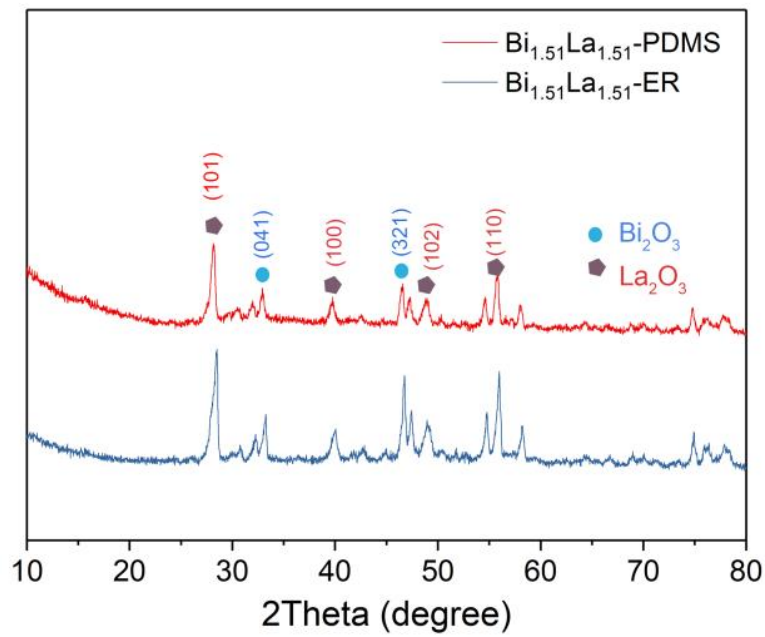

Figure S6. XRD of $\mathrm{Bi}_{1.51} \mathrm{La}_{1.51}-\mathrm{PDMS}$ and $\mathrm{Bi}_{1.51} \mathrm{La}_{1.51}-\mathrm{ER}$. 

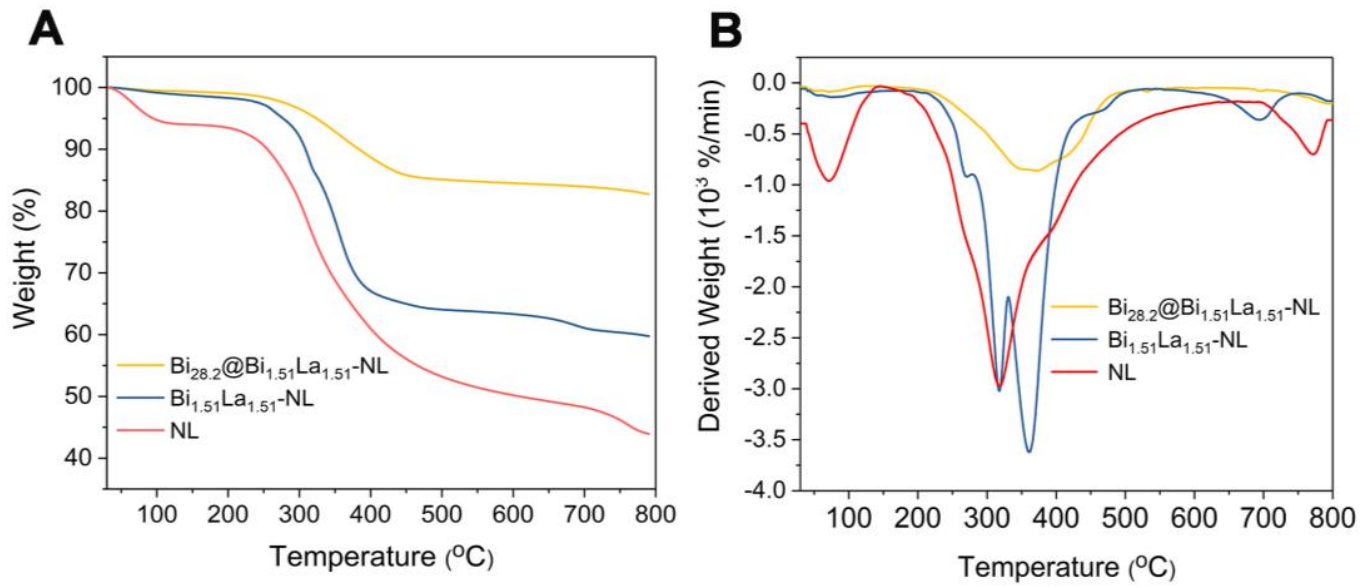

Figure S7. TGA of NL, Bi $i_{1.51} \mathrm{La}_{1.51}-\mathrm{NL}$, and $\mathrm{Bi}_{28.2} @ \mathrm{Bi}_{1.51} \mathrm{La}_{1.51}-\mathrm{NL}$. 


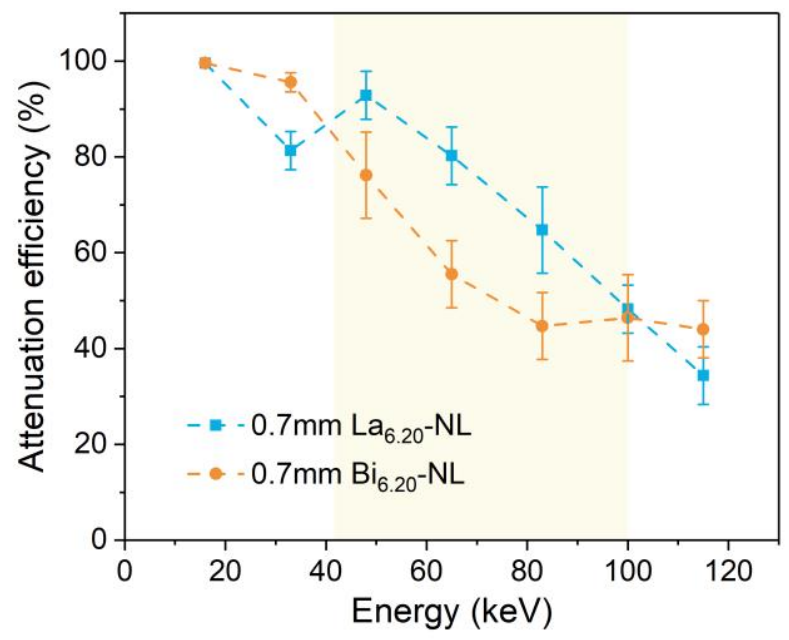

Figure S8. Attenuation efficiency of $\mathrm{Bi}_{6.20}-\mathrm{NL}$ and $\mathrm{La}_{6.20}-\mathrm{NL}$. 


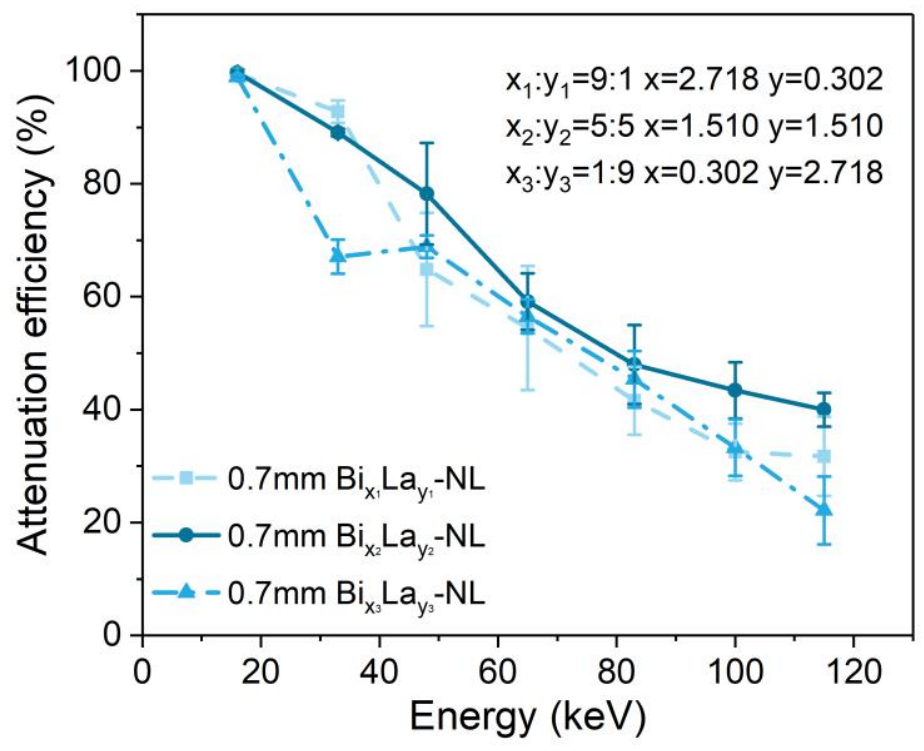

Figure S9. Attenuation efficiency of $\mathrm{Bi}_{x} \mathrm{La}_{y}-\mathrm{NL}(\mathrm{Bi}: \mathrm{La}=9: 1,5: 5,1: 9)$ with a thickness of 0.7 $\mathrm{mm}$. 


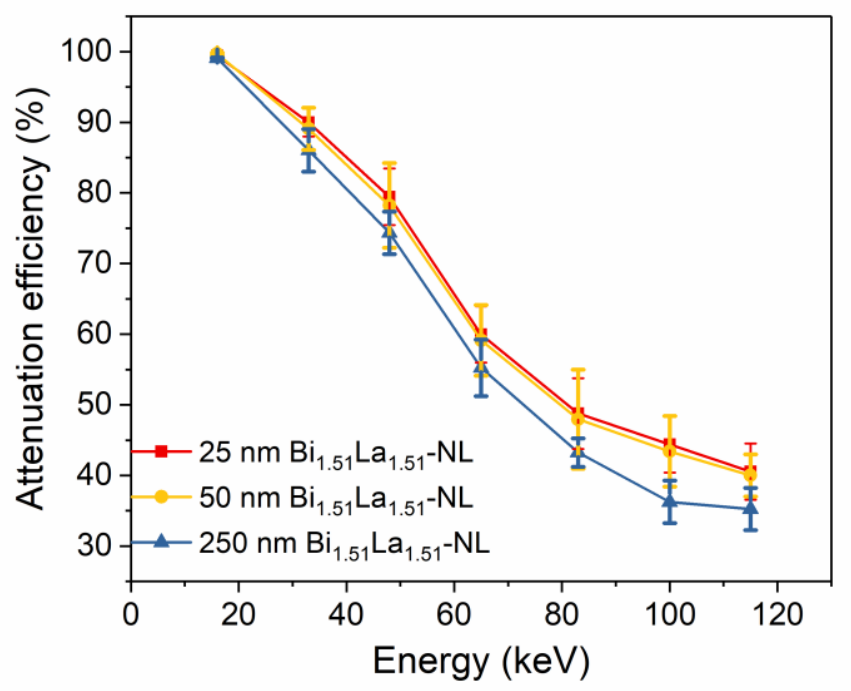

Figure S10. Attenuation efficiency of $\mathrm{Bi}_{1.51} \mathrm{La}_{1.51}-\mathrm{NL}$ with different nanoparticle sizes. 


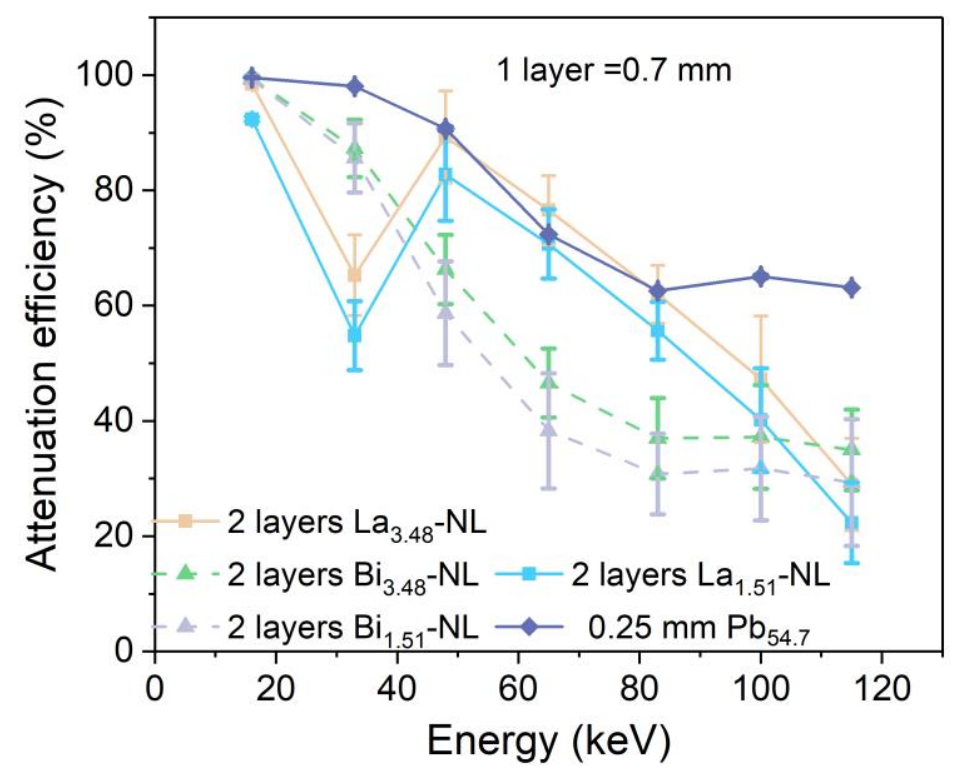

Figure S11. Attenuation efficiency of $\mathrm{La}_{3.48}-\mathrm{NL}$ (double layers), $\mathrm{Bi}_{3.48}$-NL (double layers), $\mathrm{La}_{1.51^{-}}$ $\mathrm{NL}$ (double layers), $\mathrm{Bi}_{1.51}-\mathrm{NL}$ (double layers) and $0.25 \mathrm{~mm}$ lead plate. 

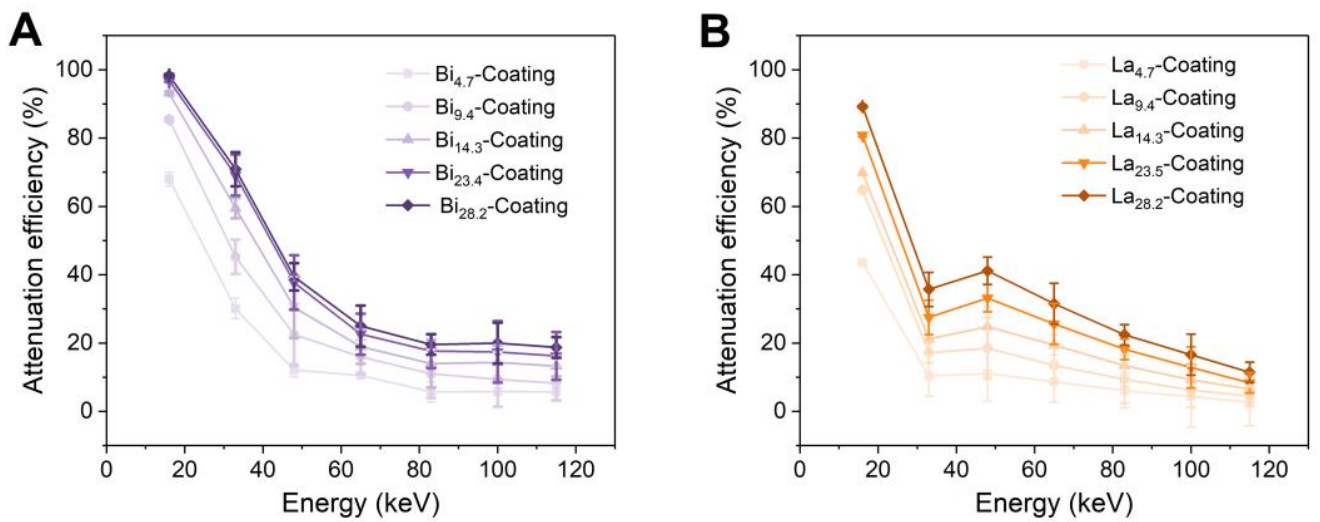

Figure S12. (A) Attenuation efficiency of $\mathrm{Bi}_{\mathrm{x}}$-Coating with different loading. ( $\mathrm{x}=4.7,9.4,14.4$, 23.5 and $28.2 \mathrm{mmol} \cdot \mathrm{cm}^{-3}$ ) (B) Attenuation efficiency of $\mathrm{La}_{\mathrm{x}}-\mathrm{Coating}$ with different loading. 


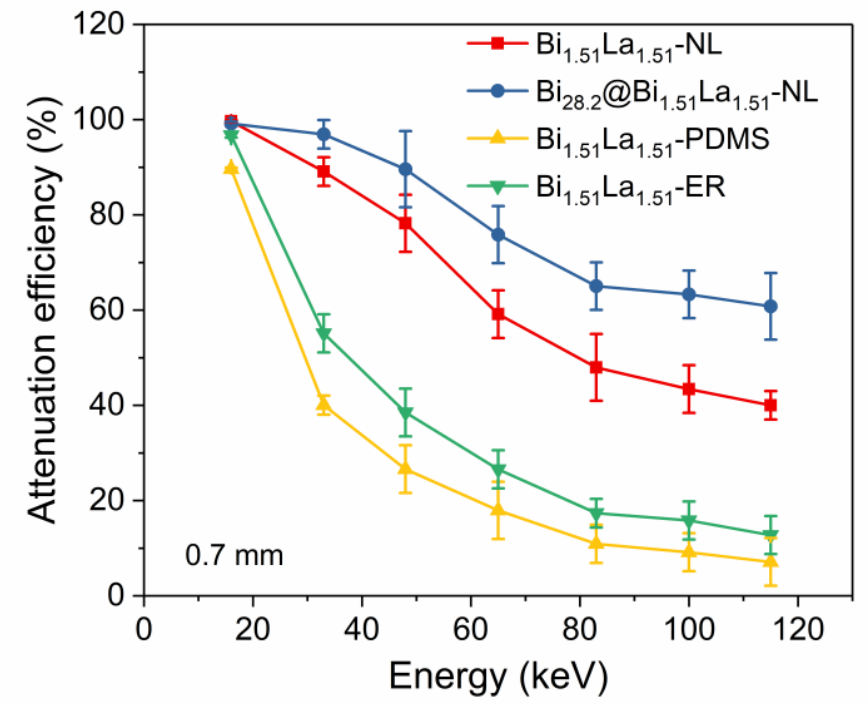

Figure S13. Attenuation efficiency of $\mathrm{Bi}_{1.51} \mathrm{La}_{1.51}-\mathrm{NL}, \mathrm{La}_{28.2} @ \mathrm{Bi}_{1.51} \mathrm{La}_{1.51}-\mathrm{NL}, \mathrm{Bi}_{1.51} \mathrm{La}_{1.51}-$ PDMS and $\mathrm{Bi}_{1.51} \mathrm{La}_{1.51}-\mathrm{ER}$. 

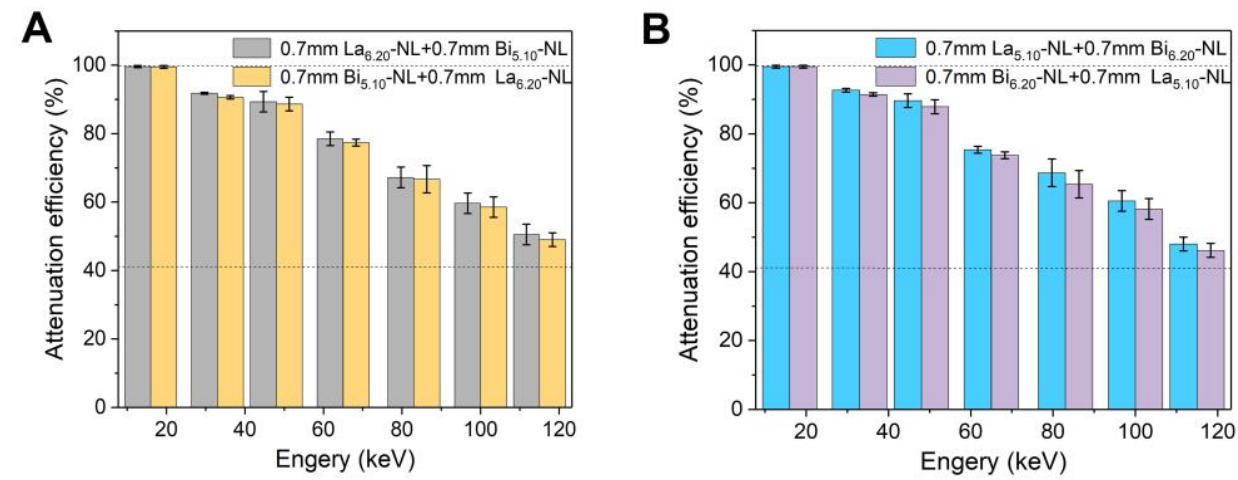

Figure S14. (A, B) Attenuation efficiency of MNPs-NL with different stacking order between Ce-NL and Bi-NL. 

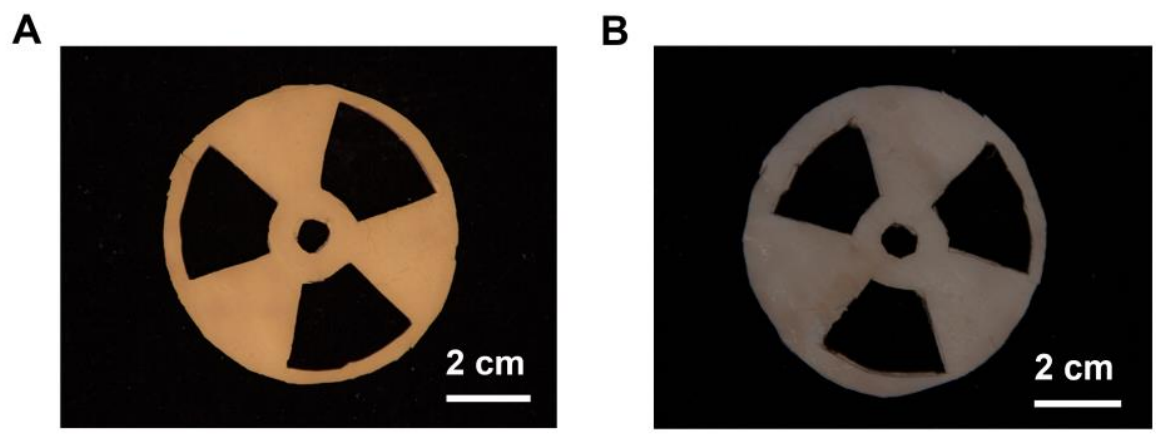

Figure S15. (A) Sample of Bi $28.2 @ \mathrm{Bi}_{3.48} \mathrm{La}_{3.48}$-NL. (B) Sample of La28.2@Bi3.48La3.48-NL. 
Table S1. Metal element concentrations of the composite materials.

\begin{tabular}{|c|c|c|c|c|}
\hline \multirow{2}{*}{ Sample } & $\mathrm{Bi}$ in matrix & La in matrix & Bi Coating & La Coating \\
\hline & \multicolumn{4}{|c|}{$\mathrm{mmol} / \mathrm{cm}^{3}$} \\
\hline $\mathrm{Bi}_{1.51-\mathrm{NL}}$ & 1.51 & & & \\
\hline $\mathrm{La}_{1.51}-\mathrm{NL}$ & & 1.51 & & \\
\hline $\mathrm{Bi}_{1.51} \mathrm{La}_{1.51}-\mathrm{NL}$ & 1.51 & 1.51 & & \\
\hline $\mathrm{Bi}_{3.48} \mathrm{La}_{3.48}-\mathrm{NL}$ & 3.48 & 3.48 & & \\
\hline $\mathrm{La}_{28.2} @ \mathrm{Bi}_{1.51} \mathrm{La}_{1.51}-\mathrm{NL}$ & 1.51 & 1.51 & & 28.2 \\
\hline $\mathrm{Bi}_{28.2} @ \mathrm{Bi}_{1.51} \mathrm{La}_{1.51}-\mathrm{NL}$ & 1.51 & 1.51 & 28.2 & \\
\hline $\mathrm{Bi}_{28.2} @ \mathrm{Bi}_{3.48} \mathrm{La}_{3.48}-\mathrm{NL}$ & 3.48 & 3.48 & 28.2 & \\
\hline $\mathrm{La}_{28.2} @ \mathrm{Bi}_{3.48} \mathrm{La}_{3.48}-\mathrm{NL}$ & 3.48 & 3.48 & & 28.2 \\
\hline
\end{tabular}


Table S2. Porosities of different materials.

\begin{tabular}{cc}
\hline Sample & Porosity \\
\hline Raw sheep skin & $81.3 \%$ \\
Tanned skin graft & $63.6 \%$ \\
$\mathrm{Bi}_{3.48} \mathrm{La}_{3.48}-\mathrm{NL}$ & $56.6 \%$ \\
$\mathrm{Bi}_{28.2} @ \mathrm{Bi}_{3.48} \mathrm{La}_{3.48}-\mathrm{NL}$ & $42.2 \%$ \\
\hline
\end{tabular}


Table S3. Attenuation coefficients of the representative materials

\begin{tabular}{cccccccc}
\hline $\begin{array}{c}\text { Energy } \\
(\mathrm{keV})\end{array}$ & $\mathrm{Pr}$ & $\mathrm{Pm}$ & $\mathrm{Sm}$ & $\mathrm{Gd}$ & $\mathrm{Tb}$ & $\mathrm{Yb}$ & $\mathrm{Lu}$ \\
\cline { 2 - 8 } & & \multicolumn{7}{c}{$\mu \mathrm{cm}^{-1}$} \\
\hline 40 & 238.68 & 116.53 & 47.83 & 54.67 & 60.00 & 62.38 & 93.18 \\
50 & 108.25 & 63.89 & 130.85 & 30.49 & 33.41 & 34.78 & 51.96 \\
60 & 67.53 & 38.99 & 83.25 & 92.83 & 100.65 & 21.74 & 32.47 \\
80 & 31.56 & 17.91 & 39.18 & 44.00 & 47.98 & 47.61 & 70.45 \\
100 & 17.47 & 9.94 & 21.81 & 261.57 & 26.75 & 26.77 & 39.56 \\
150 & 6.23 & 3.90 & 7.75 & 9.00 & 12.43 & 9.52 & 14.07 \\
200 & 3.12 & 2.02 & 3.91 & 4.35 & 4.69 & 4.76 & 6.99 \\
\hline
\end{tabular}


Table S4. Cost analysis of the prepared samples

\begin{tabular}{cc}
\hline Sample & Price \\
\hline $\mathrm{Bi}_{2} \mathrm{O}_{3} \mathrm{NPs}$ & $\$ 43 / \mathrm{kg}$ \\
$\mathrm{La}_{2} \mathrm{O}_{3} \mathrm{NPs}$ & $\$ 29 / \mathrm{kg}$ \\
Leather matrix & $\$ 17 / \mathrm{m}^{2}$ \\
$\mathrm{Bi}_{28.2} @ \mathrm{Bi}_{1.51} \mathrm{La}_{1.51}-\mathrm{NL}$ garment & $\$ 85$ \\
Lead garment & $\$ 100$ \\
\hline
\end{tabular}

